\title{
Reliability and validity of RT6 accelerometer with comparing different walking self-selected speeds
}

\author{
Neslihan Duruturk ${ }^{1 *}$, Nihan Ozunlu Pekyavas ${ }^{1}$, Aslican Caglar ${ }^{1}$ and Mustafa Agah Tekindal ${ }^{2}$ \\ ${ }^{1}$ Department of Physiotherapy, Baskent University , Turkey \\ ${ }^{2}$ Selcuk University, Department of Biostatistical, Konya, Turkey
}

\begin{abstract}
Aim of this study was to assess the reliability and validity of RT6 in healthy, young, sedentary adults. A total of 41 healthy, young, sedentary adults were recruited to this study. Sociodemographic data and IPAQwere collected from the subjects. Subjects wore RT6 accelerometer and pedometer on their pelvises. Subjects were asked walk at their normal and brisk walking speeds for 10 minutes. These activities were repeated on a treadmill using the individual speeds from normal ground walking on two settings 1 week apart. Bland-Altman analyses were performed to determine the mean difference and the levels of agreement. The Cronbach's alpha coefficient of the questionnaire was used. There was a $63.2 \%$ agreement between the treadmill and the normal ground at normal speed measurements. There was no statistically significant correlation between the pedometer and other measures. This study found that RT6 is a valid measurement tool at normal and active walking speeds in measurement of physical activity.
\end{abstract}

\section{Introduction}

Physical activity is very important in prevention of diseases and increasing quality of life. There is evidence that regular physical activity contributes to the primary and secondary prevention of several chronic diseases. The greatest improvements in health status are stated to be are seen when people who are least fit become physically active [1].

Physical activity level may be assessed by many methods including both objective and subjective methods. The International Physical Activity Questionnaire (IPAQ) is one of the most widely used questionnaires for physical activity [2,3]. The short version of the IPAQ has been tested extensively and is now used in many international studies [4]. Turkish versions of the IPAQ short and long forms are found reliable and valid in assessment of physical activity [5]. Although it's wide usage, a review about the validity of IPAQ- Short form state that the IPAQ-Short form typically overestimated physical activity as measured by objective criterion by an average of 84 percent. They also state that the evidence to support the use of the IPAQ-SF as an indicator of relative or absolute physical activity is weak [6].

Pedometers are small, relatively inexpensive devices worn at the hip to count the number of steps walked per day. Although there is not detailed evidence of their effectiveness, they have recently experienced a surge in popularity as a tool for motivating and monitoring physical activity [7].

Accelerometer is a popular, reliable, non-invasive and low cost method for objective physical activity assessment [8]. The accelerometer determines body acceleration presented as an analogue voltage created by a piezoelectric instrument that is sensitive to compression in a vertical direction. The signal is then summarized over a userdefine time into counts. The numbers of counts determine the level of physical activity as higher counts refer to higher physical activity level. It can also be used to determine the pattern of movement in X, $\mathrm{Y}$ and $\mathrm{Z}$ axis [9]. The RT6 triaxial accelerometer (Research Tracker 6, Version 3.1, StayHealthy, Inc., Monvoria, CA, USA) is a new model of
RT3 accelerometer which is a reliable accelerometer for measurement of physical activity in different conditions including walking and running at standardized speeds on a treadmill [10-12]. The RT6 is also a waist-mounted device used to assess free-living physical activity. But still, no evidence is met about the reliability and validity of RT6 in the literature. Therefore, our aim for this study was to assess the reliability and validity of RT6 in healthy, young, sedentary adults.

\section{Methods}

\section{Sample size justification}

The power analysis indicated that 33 participants were needed with $80 \%$ power and a $5 \%$ type 1 error. We increased the primary sample size by $20 \%$ to avoid the loss of potential non-respondents and our minimum sample size was defined as 41 subjects for the study. The study was approved by the Baskent University Institutional Review Board and Ethics Committee (Project number: KA16/247).

\section{Participants}

The study was performed at Baskent University, Faculty of Health Sciences, Department of Physiotherapy and Rehabilitation, Ankara, Turkey. A total of 41 healthy, young, sedentary adults were recruited to our study. The inclusion criteria for our study were; 1 . to be in good health, 2. to be able to walk independently for 60 minutes at their selfselected speeds, 3 . between 18 and 25 years old, 4 .to be able to attend the initial and follow-up session. The exclusion criteria were; 1 . any history of current or past medical conditions that prevent them from

Correspondence to: Duruturk Neslihan, Faculty of Health Sciences, Department of Physiotherapy and Rehabilitation, Başkent University, Turkey, E-mail: nalkan@baskent.edu.tr

Key words: physical activity, accelerometer, RT6, reliability, validity

Received: October 24, 2017; Accepted: November 27, 2017; Published: November 30, 2017 
walking independently for 60 minutes, 2 . to be unable to understand written and verbal instructions, and 3 . to be unable to provide written informed consent.

\section{Instrumentation}

Physical activity was evaluated by both objective (accelerometer, pedometer) and subjective (IPAQ) methods. The RT6 accelerometer provides an objective measurement for physical activity. The RT6 accelerometer assesses acceleration in each anatomical axis with vertical ( $\mathrm{x})$, anteroposterior $(\mathrm{y})$, and mediolateral $(\mathrm{z})$ assessments. The square-root of the sum of squared accelerations for each axis provides a vector magnitude $(\mathrm{VM})$ in counts per minute (counts/min). The RT6 accelerometer measures acceleration periodically and converts it to a digital representation, which is then processed to obtain an "activity count" and stored in the memory. The exact relationship of the activity count to the acceleration (measured in meters per second squared or $\mathrm{g}$, where $1 \mathrm{~g}=9.81 \mathrm{~m} / \mathrm{s} 2)$ is not stated clear $[10,13]$.

Pedometer is one of the tools for evaluating, increasing and encouraging physical activity of an individual. The TNV Pedometer (PM 2000, Zhongshan Tender Electric Appliance Co, Ltd, China) was selected in this study. Every time the hip moves up and down during normal walking motion, the internal mechanism also moves, which in turn causes the pedometer to record a step. So, the pedometer counts and records the steps [14].

The IPAQ is one of the most widely used questionnaires for physical activity [2,3]. Subjects were asked to fill the short form of the Turkish version [5] and data were collated and presented both as median minutes per week or median metabolic equivalent of task (MET)-minutes per week and as categorical cut point values (defined as moderate, vigorous, walking activity and total scores) following the guidelines for data processing and analysis outlined in the IPAQ (http:// www.ipaq.ki.se/scoring.pdf). The questionnaire was administered immediately after the accelerometer measurement.

\section{Procedure}

The procedures were explained to the subjects and a written consent form was obtained from all subjects prior to the study. Measurements were performed by the same physiotherapist. All subjects were tested in self-selected footwear and clothing that is comfortable for them and was asked to use the same footwear for both ground and treadmill walking to minimize possible gait-pattern changes. Sociodemographic data as age, height, weight and gender was collected from the subjects. These parameters were recorded to the subjects' profile on the RT6 accelerometer using Stay Healthy, Inc, software (Research Tracker 6, Version 3.1, Stay Healthy, Inc, Monvoria, CA, USA). Data was collected from "Data Management" total calories and activity count (VM; X axis, $\mathrm{Y}$ axis, $\mathrm{Z}$ axis).

The RT6 accelerometer was attached to the right lateral pelvis by placing it on the subject's trousers or skirt with its own plastic clip $[3,10,13]$. The pedometer was attached to the left pelvis by placing it on the subject's trousers or skirt with its own plastic clip. With the RT6 accelerometer and pedometer attached and turned on, each subject was instructed to walk for 10 minutes at a 30 -meter corridor at their self-selected normal speed, then for 10 minutes at their self-selected active speed. The subjects performed each task individually with a 5 minute seated rest between tasks. Normal speed is defined as "walking at your normal pace as if you were walking to a friend's house" and active speed is defined as "walking at your brisk pace as if you were late for an appointment or lecture" [10]. The mean walking speeds for both the normal and active speeds (kilometres per hour) using the distance walked in for 10 minutes on the ground $(X=V \times t)$. This calculation was done in order to standardize the self-selected normal and active speeds to be used at the treadmill.

After 30 minutes of rest period, subjects walked on the treadmill for 10 minutes at normal speed and 10 minutes at active speed. The subjects performed each task individually with a 5 minute seated rest between tasks. At the conclusion of each subject's trial, RT6 accelerometer was removed from the subject's hip and placed it in the "docking station" to upload data into the Stay Healthy, Inc, software.

A week later, subjects returned for the re-test application to repeat the two treadmill tasks (10 minutes at normal speed and 10 minutes at active speed with a 5 minute rest between tasks). Each subject also wore the same RT6 as the first assessment with the same attachment. Health statuses of the subjects were asked not to have any change. Same applications were used as the first assessment for re-test procedure.

\section{Statistical analysis}

Total of six 10-minute walking settings (two ground and four treadmill) per participant $(n=41)$ were used for analysis in this study. For discrete and continuous variables, descriptive statistics (mean, standard deviation, median, minimum value, maximum value, and percentile) were given. Bland-Altman analysis were performed to determine the mean difference and the levels of agreement $( \pm 2$ standard deviation [SD]) for vector magnitudes between walking on the treadmill and ground at the two walking speeds on first testing day and retest days in this study. Two continuous variables, the relationship between Pearson Correlation Coefficient In the case of providing the prerequisites for parametric tests were evaluated by Spearman correlation coefficient. The Cronbach's alpha coefficient of the survey is used. IBM Corp. Released 2012. IBM SPSS Statistics for Windows, Version 21.0. Armonk, NY: IBM Corp was used for all statistical analyses.

\section{Results}

A total of 41 sedentary young adults (19 males and 22 females) were recruited from Baskent University, Faculty of Health Sciences, Department of Physiotherapy and Rehabilitation. Table 1 presents the demographics and walking characteristics of the participants. Table 2 presents the VM descriptive values for participants walking on the treadmill and normal ground.

The way to calculate the reliability coefficient depends on the type of the variables, the source, and the number of applications. The variability of the calculation path also changes the interpretive meaning of the reliability coefficient. The reliability coefficient is the degree of clearance from random faults and gives the amount of error involved in the measurement results. Reliability is required to take values ranging from 0 to +1 , and to obtain values close to +1 . A reliability coefficient of more than 0.60 is a desirable result. Cronbach alpha reliability of the measurement tool that each scale ( $\alpha$ ) reliability, there is a sense of reliability in terms of internal consistency. The Cronbach's alpha ( $\alpha$ ) coefficient of the survey used in the survey is shown in the Table 3.

According to Cronbach's alfa coefficients (Table 3) there is a $63.2 \%$ agreement between the treadmill and the normal ground at normal speed measurements; $62.1 \%$ agreement between the treadmill and the normal ground at brisk speed; moderate degree of $52.4 \%$ agreement between treadmill and retest treadmill ground at normal speed measurements and moderate degree of $56.6 \%$ agreement between treadmill and normal ground at bris speed measurements. 
Table 1. Subjects demographic and walking characteristics

\begin{tabular}{|l|l|}
\hline \multicolumn{1}{|c|}{ Mean \pm SD } \\
\hline Age (years) & $24.26 \pm 5.36$ \\
\hline BMI $\left(\mathbf{k g} / \mathbf{m}^{2}\right)$ & $22.08 \pm 3.10$ \\
\hline Normal walking speed $(\mathbf{k m} / \mathbf{h})$ & $6.95 \pm 2.04$ \\
\hline Brisk walking speed $(\mathbf{k m} / \mathbf{h})$ & $880.27 \pm 257.33$ \\
\hline Normal walking pedometer step counts & $1081.951 \pm 130.68530$ \\
\hline Brisk walking pedometer step counts & $1230.29 \pm 143.83$ \\
\hline IPAQ total score & $2494.35 \pm 1949.65$ \\
\hline
\end{tabular}

BMI: body mass index, kg: kilograms, m: meter, h: hours, IPAQ: International Physical Activity Questionnaire, SD: standard deviation

Table 2. Descriptive data for vector magnitudes for normal ground and treadmill.

\begin{tabular}{|l|c|c|c|c|}
\hline & Minimum & Maximum & Mean & $\begin{array}{c}\text { Std. } \\
\text { Deviation }\end{array}$ \\
\hline VM- normal ground- normal speed & 103.98 & 293.48 & 188.02 & 54.89 \\
\hline VM- normal ground- brisk speed & 154.50 & 369.41 & 230.37 & 43.39 \\
\hline VM- treadmill- normal speed & 102.87 & 321.78 & 200.88 & 53.85 \\
\hline VM- treadmill- brisk speed & 184.54 & 412.80 & 248.58 & 40.87 \\
\hline VM- retest treadmill- normal speed & 100.12 & 301.85 & 226.62 & 55.39 \\
\hline VM- retest treadmill- brisk speed & 164.20 & 361.05 & 277.22 & 44.77 \\
\hline
\end{tabular}

(VM: vector magnitude.)

Table 3. Cronbach's alfa coefficients between normal ground and treadmill.

\begin{tabular}{|l|l|c|}
\hline & & $\begin{array}{c}\text { Cronbach's } \\
\text { Alpha values }\end{array}$ \\
\hline VM- normal ground- normal speed & VM- treadmill- normal speed & 0.632 \\
\hline VM- treadmill- normal speed & VM- retest treadmill- normal speed & 0.524 \\
\hline VM- normal ground- brisk speed & VM- treadmill- brisk speed & 0.621 \\
\hline VM- treadmill- brisk speed & VM- retest treadmill- brisk speed & 0.565 \\
\hline
\end{tabular}

(VM: vector magnitude.)

Bland-Altman plots show that mean difference between two measures and dottedlines indicate $95 \%$ confidence interval (CI) $( \pm 1.96$ standard deviation) about mean agreement at the first testing days and retest days on normal ground and treadmill ( Figures 1-4).

If the differences are normal distributions, it is expected that the differenc - will be ra lomly distributed around zero and $95 \%$ will be between ${ }^{2}-1.96 \mathrm{~s}$ and ${ }^{2}+1.96 \mathrm{~s}$. In this situation, it can be said that th is no relation between averages and differences. In this method, ${ }^{d}{ }_{ \pm}$ $1.96 \mathrm{~s}$ is called "compliance limits". Table 4 presents agreement values of vector magnitude according to Bland Altman method. We can state that the test is valid according to the results because the analyses were between the limits of confidence.

Table 5 presents correlations between pedometer (step counts) and accelerometer (vector magnitude). There is no statistically significant correlation between the pedometer and other measures.

\section{Discussion}

In this study we investigated the reliability and validity of RT6 in healthy young sedentary adults and found that RT6 is a valid measurement tool at normal and active walking speeds in measurement of physical activity.

Investigating the validity of RT6, our method included two measurements (test and retest) which is an empowering factor for reliability. A similar study investigating the construct validity of RT3 accelerometer in comparison of level-ground and treadmill walking at self-selected speeds found that measurement and discrimination of walking intensity employing RT3 accelerometer VM counts/min on the treadmill demonstrated reasonable validity and stability over two time points compared with level-ground walking [10].
There are many pedometers sold on markets worn indifferently either at the waist, wrist, or as a necklace. A study investigating pedometer's accuracy according to their position worn at various walking speeds showed that at all positions, pedometers generated significant errors at slow speeds and therefore cannot be used reliably to evaluate the amount of physical activity for people walking slower than $0.6 \mathrm{~m} / \mathrm{s}(2.16 \mathrm{~km} / \mathrm{h}$, or $1.24 \mathrm{mph})$ [15]. Regardless of that, pedometers are used in many areas such as primary care [16] increasing physical activity and stress management $[17,18]$ decreasing cardiovascular risk factors [19] and adolescents [20]. RT6 and pedometer results were not found significantly correlated in our study. The pedometer used in our study is a $2 \mathrm{D}$ measurement tool which only counts the steps while walking. RT6 is a $3 \mathrm{D}$ measurement tool and is able to give 3 axes information not only while walking but also in every physical movement. Therefore, RT6 may be considered more objective as a measurement tool for physical activity.

IPAQ is used in many recent studies to measure physical activity level of post-menopausal women [21] breast cancer patients (Canário, et al,1992), cardiovascular disease patients [22] and diabetes $[23,24]$. Turkish versions of the IPAQ short and long forms are found reliable and valid in assessment of physical activity [5]. Cerin et al. examined agreement between self-reported (IPAQ-Long Form) and accelerometer-based estimates of physical activity and sedentary behaviour across six countries and identified correlates of between-method agreement [25]. They showed that after adjusting for sociodemographic and behavioural factors, the absolute disagreement

Table 4. Agreement values of vector magnitude according to Bland Altman method.

\begin{tabular}{|l|l|c|c|c|c|}
\hline & \multicolumn{2}{|c|}{$\overline{\boldsymbol{d}}$} & $\overline{\boldsymbol{d}} \pm \mathbf{1 . 9 6}$ sd & \multicolumn{1}{|c|}{$\mathbf{p}$} \\
\hline $\begin{array}{l}\text { VM- normal ground- } \\
\text { normal speed }\end{array}$ & $\begin{array}{l}\text { VM- treadmill- normal } \\
\text { speed }\end{array}$ & -12.9 & 97.7 & -123.4 & $0.001 * *$ \\
\hline $\begin{array}{l}\text { VM- normal ground- } \\
\text { brisk speed }\end{array}$ & $\begin{array}{l}\text { VM- treadmill- brisk } \\
\text { speed }\end{array}$ & -18.4 & 52 & -88.8 & $0.001 * *$ \\
\hline $\begin{array}{l}\text { VM- treadmill- normal } \\
\text { speed }\end{array}$ & $\begin{array}{l}\text { VM- retest treadmill- } \\
\text { normal speed }\end{array}$ & -25.7 & 95.8 & -147.3 & $0.001 * *$ \\
\hline $\begin{array}{l}\text { VM- treadmill- brisk } \\
\text { speed }\end{array}$ & $\begin{array}{l}\text { VM- retest treadmill- } \\
\text { brisk speed }\end{array}$ & -28.6 & 63.8 & -121.1 & $0.001 * *$ \\
\hline
\end{tabular}

$(* * \mathrm{p}<0.01$, sd: standard deviation, VM: vector magnitude)

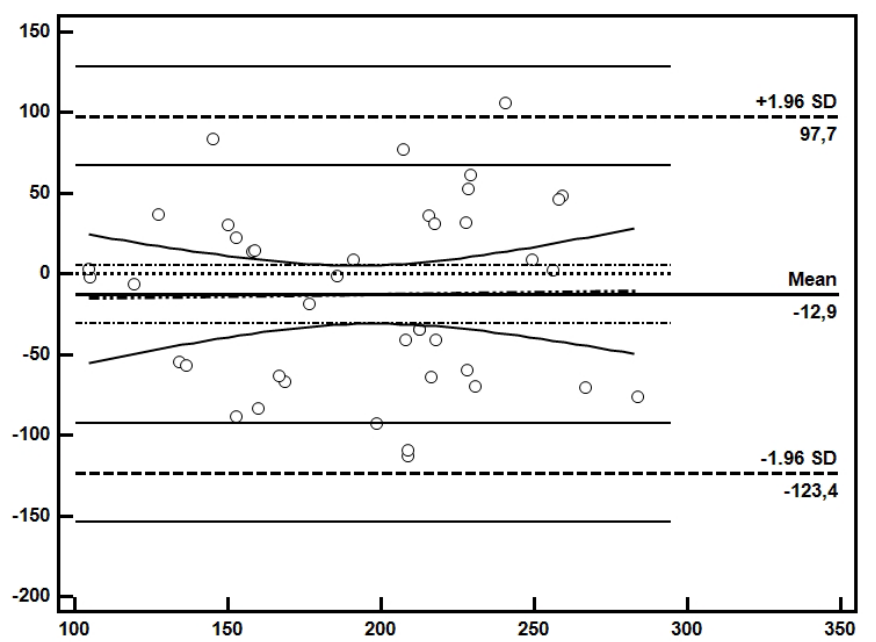

Figure 1. Bland-Altman plot of changes in participants' normal speed (normal ground walking vs treadmill walking) at same set speed on first testing day.Solid line indicates mean difference between two measures and dottedlines indicate $95 \%$ Confidence Interval (CI) ( \pm 1.96 standard deviation) about mean agreement. Data points above and below CI were considered outliers. 
Table 5. Correlations between pedometer (step counts) and accelerometer (vector magnitude).

\begin{tabular}{|c|c|c|c|c|c|}
\hline & & $\begin{array}{l}\mathrm{VM} \text { in normal speed- normal } \\
\text { ground }\end{array}$ & $\begin{array}{l}\text { VM in brisk speed- normal } \\
\text { ground }\end{array}$ & VM in normal speed- treadmill & VM in brisk speed- treadmill \\
\hline \multirow{2}{*}{ Step counts in normal speed- normal ground } & $\mathbf{r}$ & -0.260 & - & - & - \\
\hline & $\mathbf{p}$ & 0.101 & - & - & - \\
\hline \multirow{2}{*}{ Step counts in brisk speed- normal ground } & $\mathbf{r}$ & - & -0.088 & - & - \\
\hline & $\mathbf{p}$ & - & 0.586 & - & - \\
\hline \multirow{2}{*}{ Step counts in normal speed- treadmill } & $\mathbf{r}$ & - & - & 0.104 & - \\
\hline & $\mathbf{p}$ & - & - & 0.517 & - \\
\hline \multirow{2}{*}{ Step counts in brisk speed- treadmill } & $\mathbf{r}$ & - & - & - & -0.124 \\
\hline & $\mathbf{p}$ & - & - & - & 0.442 \\
\hline
\end{tabular}

(VM: vector magnitude.)

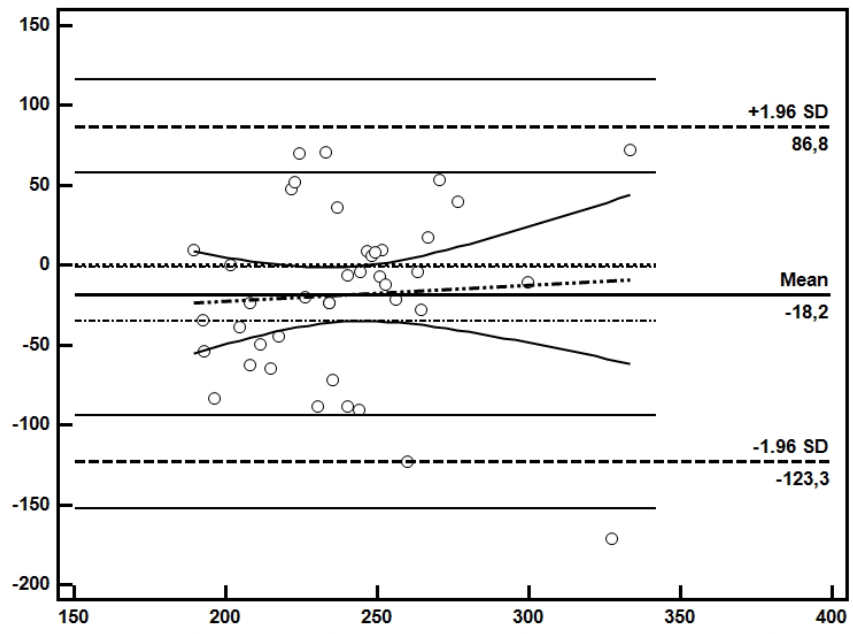

Figure 2. Bland-Altman plot of changes in participants' brisk speed (normal ground walking vs treadmill walking) at same set speed on first testing day. Solid line indicates mean difference between two measures and dotted lines indicate $95 \%$ Confidence Interval (CI) ( \pm 1.96 standard deviation) about mean agreement. Data points above and below $\mathrm{CI}$ were considered outliers.

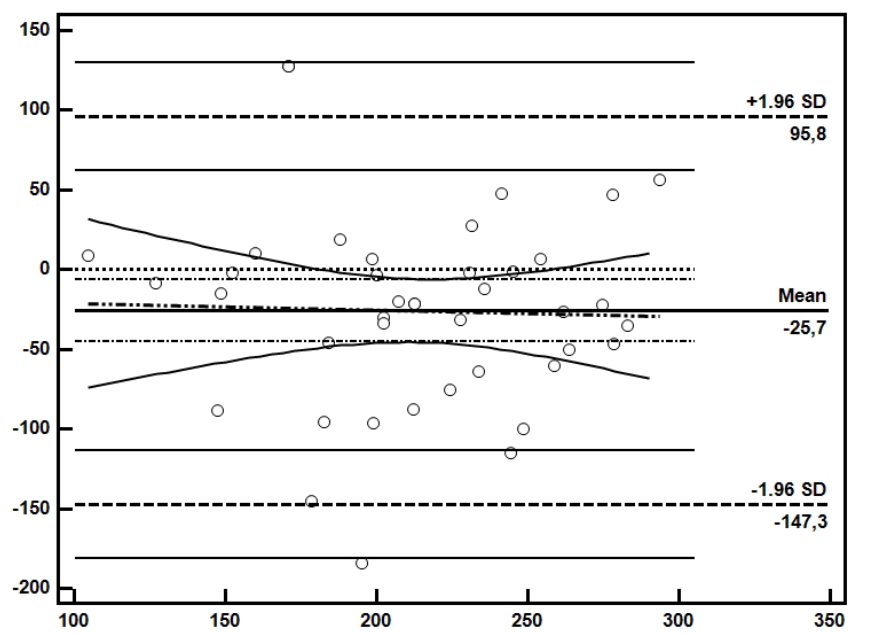

Figure 3. Bland-Altman plot of changes in participants' normal speed (normal ground walking vs treadmill walking) at same set speed from first testing day to re-test day. Solid line indicates mean difference between two measures and dotted lines indicate $95 \%$ Confidence Interval (CI) ( \pm 1.96 standard deviation) about mean agreement. Data points above and below CI were considered outliers.

between pairs of IPAQ-Long Form and accelerometer-based physical activity variables remained significantly different across cities/ countries. There was found systematic cultural and/or linguistic and

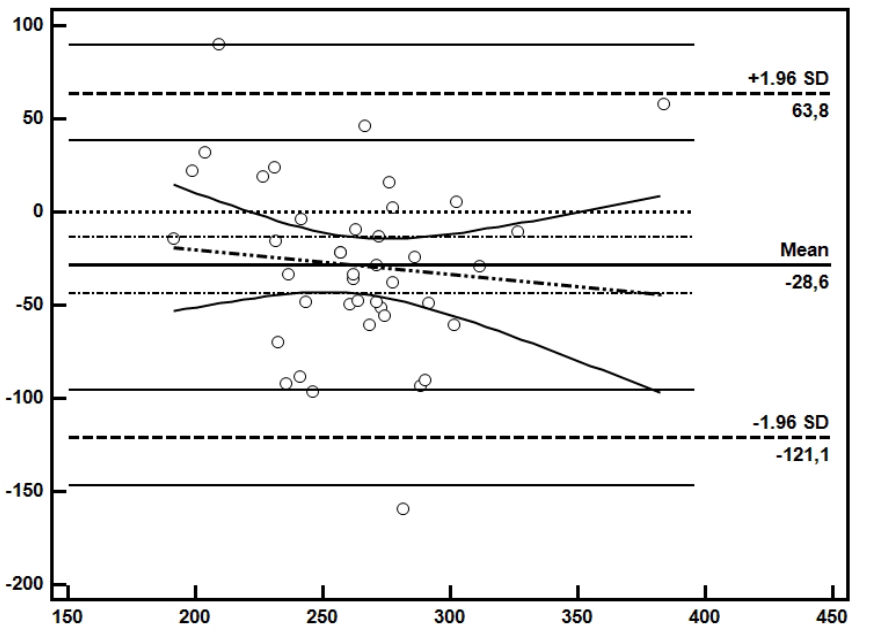

Figure 4. Bland-Altman plot of changes in participants' brisk speed (normal ground walking vs treadmill walking) at same set speed from first testing day to re-test day. Solid line indicates mean difference between two measures anddotted lines indicate $95 \%$ Confidence Interval (CI) ( \pm 1.96 standarddeviation) about mean agreement. Data points above and below CI were considered outliers.

sociodemographic differences in absolute agreement between the IPAQ-Long Form and the accelerometer-based physical activity and sedentary behaviour variables [25]. Considering the results of IPAQ and RT6, IPAQ results were not found significantly correlated to RT6 results in our study. IPAQ is a questionnaire for measurement of physical activity and depends on the answers given by subjects, so it may be considered more subjective than RT6. RT6 gives the results of vector magnitude which refers to the $3 \mathrm{D}$ movement in all axes which may be more objective in measuring physical activity. As there is no correlation found between IPAQ, accelerometer and pedometer, this may show us that these three measurement tools should not be used together through an assessment.

The way to calculate the reliability coefficient depends on the type of the variables, the source, and the number of applications. The variability of the calculation path also changes the interpretive meaning of the reliability coefficient. The reliability coefficient is the degree of clearance from random faults and gives the amount of error involved in the measurement results. Reliability is required to take values ranging from 0 to +1 , with values closer to +1 . A reliability coefficient of more than 0.60 is a desirable result. There is a reliability of Cronbach alpha $(\alpha)$ reliability and internal consistency when the measurement tool is scaled each time. At our study we found that there is a $63.2 \%$ agreement between the treadmill and the normal ground at normal speed measurements; $62.1 \%$ agreement between the treadmill and the normal ground at brisk speed; moderate degree of $52.4 \%$ agreement 
between treadmill and retest treadmill ground at normal speed measurements and moderate degree of $56.6 \%$ agreement between treadmill and normal ground at bris speed measurements.

According to the Bland Altman method, if the differences have normal distribution, it is expected that the differences will be randomly distributed around zero and 95\% will be between " $\mathrm{d}$ A $-1,96 \mathrm{~s}$ and $\mathrm{d}$ $+1,96 \mathrm{~s}$ ". In this situation, it can be said that there is no correlation between averages and differences $(1,4)$. In this method, $\mathrm{d}^{-} \pm 1,96 \mathrm{~s}$ is called "agreement limits" [26]. We can state that the test is valid according to the results because the analyses were between the limits of confidence.

Bland-Altman plot of changes in participants' normal speed and brisk speed show similar results between the normal ground and the treadmill (almost all analyses are within 95\% confidence limits). However, looking at the treadmill Bland-Altman plots of retest results, a moderate agreement appears. Analyses are very close to the limit values in the confidence interval.

Physical activity has been focused in last decade and has a major role in prevention of chronic diseases. Physical activity is defined as any bodily movement produced by skeletal muscles that require energy expendit [27]. Physiotherapists play an important role on increasing physical activity which can reduce the risk of cardiovascular diseases, diabetes, colon and breast cancer, and depression. Moreover, adequate levels of physical activity will decrease the risk of a hip or vertebral fracture and help control weight [27]. Increasing physical activity should be one of the most important tasks of all health care workers and RT6 may be a valid and reliable tool in their toolbox.

\section{References}

1. Warburton DER, Nicol CW, Bredin SSD (2006) Health benefits of physical activity: the evidence CMAJ 174: 801-809. [Crossref]

2. Poppel MN, Chinapaw MJ, Mokkink LB (2010) Physical activity questionnaires for adults: a systematic review of measurement properties. Sports Med 40: 565-600. [Crossref]

3. Hernandez-Hernandez V, Ferraz-Amaro I, Diaz-Gonzalez F (2014) Influence of disease activity on the physical activity of rheumatoid arthritis patients. Rheumatology 53:722-731. [Crossref]

4. Hagstromer M, Oja P, Sjostrom M (2005) The International Physical Activity Questionnaire (IPAQ): a study of concurrent and construct validity Public Health Nutr. 9: 755-762. [Crossref]

5. Saglam M, Arikan H, Savci S, (2010) International physical activity questionnaire: reliability and validity of the Turkish version Percept Mot Skills 111: 278-284. [Crossref]

6. Lee PH, Macfarlane DJ, Lam TH, Stewart MS (2011) Validity of the international physical activity questionnaire short form (IPAQ-SF): A systematic review Int J Behav Nutr Phys Act.8: 115. [Crossref]

7. Bravata DM, Smith-Spangler C, Sundaram V (2007) Using pedometers to increase physical activity and improve health: a systematic review JAMA.21: 2296-2304. [Crossref]

8. Toschke JA, von Kries R, Rosenfeld E, Tosche AM (2007) Reliability of physical activity measures from accelerometry among preschoolers in free-living conditions Clin Nutr 26: 416-420. [Crossref]
9. Vanhelst J, Béghin L, Duhamel A (2012) Comparison of uniaxial and triaxial accelerometry in the assessment of physical activity among adolescents under freeliving conditions: the HELENA study BMC Med Res Methodol.12: 26-32. [Crossref]

10. Hendrick P, Boyd T, Low O (2010) Construct validity of RT3 accelerometer: A comparison of level-ground and treadmill walking at self-selected speeds $J$ Rehabil Res Dev.47: 157-168. [Crossref]

11. Hussey J, Bennett K \& Dwyer JO (2009) Validation of the RT3 in the measurement of physical activity in children J Sci Med Sport. 2: 130-133. [Crossref]

12. Rowlands AV, Thomas PW, Eston RG, Topping R (2004) Validation of the RT3 triaxial accelerometer for the assessment of physical activity Med Sci Sports Exerc. 36: 518-524. [Crossref]

13. Powell SM, Jones DI, Rowlands AV (2003) Technical variability of the RT3 accelerometer. Med Sci Sports Exerc. 35: 1773-1778. [Crossref]

14. Schneider PL, Crouter SE, Bassett D R (2004) Pedometer measures of free Living physical activity: Comparison of 13 models Med Sci Sports Exerc.36: 331-335. [Crossref]

15. Ehrler F, Weber C, Lovis C (2016) Influence of Pedometer Position on Pedometer Accuracy at Various Walking Speeds: A Comparative Study J Med Internet Res. 6 : e268. [Crosref]

16. Pears S, Bijker M, Morton K (2016) A randomised controlled trial of three very brief interventions for physical activity in primary care BMC Public Health 30: 1033. [Crossref]

17. Razani N, Kohn MA, Wells NM , Thompson D, Hamilton Flores H, et al. (2016) Design and evaluation of a park prescription program for stress reduction and health promotion in low-income families: The Stay Healthy in Nature Everyday (SHINE) study protocol Contemp Clin Trials. 29: 8-14. [Crossref]

18. Gába A, Cuberek R, Svoboda Z Chmelík F, Pelclová J, et al. (2016) The effect of brisk walking on postural stability, bone mineral density, body weight and composition in women over 50 years with a sedentary occupation: a randomized controlled trial $B M C$ Womens Health 21: 63. [Crossref]

19. Tudor-Locke C, Schuna JM Jr, Han H, Aguiar EJ, Green MA, et al. (2016) Step-based Physical Activity Metrics and Cardiometabolic Risk: NHANE Med Sci Sports Exerc. 2005-2006. [Crossref]

20. Suchert V, Steinvoord K, Schwarz J, Isense B, Krieger C (2016) Motivational Aspects and Critical Issues of a Pedometer Intervention in Adolescents Health Promot Pract. pii: 1524839916667273. [Crossref]

21. Dallanezi G, Freire BF, Nahás EA, Nahás-Neto J, Corrente JE, et al. (2016) Physical Activity Level of Post-menopausal Women with Low Bone Mineral Density Rev Bras Ginecol Obstet. 38: 225-230. [Crossref]

22. Czeczelewska E, Czeczelewski J, Wasiluk A, Saczuk J (2016) Evaluation of the Usability of Selected Questionnaires Assessing Physical Activity in the Prophylaxis of Cardiovascular Diseases Adv Clin Exp Med. 25:59-67. [Crossref]

23. Brugnara L, Murillo S, Novials A, Rojo-Martínez G, Sorigue R, et al. (2016) Low Physical Activity and Its Association with Diabetes and Other Cardiovascular Risk Factors: A Nationwide, Population-Based Study PLoS One 11: e0160959. [Crossref]

24. Praidou A, Harris M, Niakas D, Labiris G (2016) Physical activity and its correlation to diabetic retinopathy $J$ Diabetes Complications 31:456-461 [Crossref]

25. Cerin E, Cain K, Oyeyemi AL, Owen N, Conway TL, et al. (2016) Correlates of Agreement between Accelerometry and Self-reported Physical Activity Med Sci Sports Exerc.48: 1075-1084. [Crossref]

26. Bland JM, Altman DG (1986) Statistical methods for assessing agreement between two methods of clinical measurement Lancet I: 307-310. [Crossref]

27. http://www.who.int/topics/physical_activity/en/

Copyright: (C2017 Duruturk N. This is an open-access article distributed under the terms of the Creative Commons Attribution License, which permits unrestricted use, distribution, and reproduction in any medium, provided the original author and source are credited. 